\title{
Temporary central venous catheter at hemodialysis initiation and reasons for use: a cross-sectional study
}

Izaya Nakaya* (D, Taijiro Goto, Yuki Nakamura, Kazuhiro Yoshikawa, Junji Oyama, Yoshihiko Tamayama, Mizuho Morooka, Sadatoshi Ito, Hirotaka Ishioka, Yuki Matsuura and Jun Soma

\begin{abstract}
Background: Creating permanent vascular access (VA) is recommended before hemodialysis initiation in patients with end-stage renal disease (ESRD). Although many patients are still introduced to hemodialysis with temporary central venous catheters (CVCs), the reasons for their use remain unclear. We aimed to clarify the characteristics of Japanese patients introduced to hemodialysis using temporary CVCs, the reasons for their use, and whether this rate can be reduced in the future.
\end{abstract}

Methods: We conducted this cross-sectional study in an acute care general hospital in Japan. We enrolled 393 patients aged $\geq 18$ years who received a permanent VA creation for initiating hemodialysis. We classified participants into the temporary CVC group or the permanent VA group according to the VA type at hemodialysis initiation and compared their backgrounds. We identified why permanent VA could not be used at hemodialysis initiation for patients in the temporary CVC group.

Results: Of the 393 patients, 137 (35\%) initiated hemodialysis with a temporary CVC, and arteriovenous fistulas (AVFs) were created as the first VA in all patients during hospitalization following hemodialysis initiation. The remaining 256 patients (65\%) initiated hemodialysis via AVF cannulation. The duration of predialysis nephrology care was significantly shorter in the temporary CVC group than that in the permanent VA group. The median time from AVF creation to the first successful cannulation was also shorter in the temporary CVC group (8 vs. 66 days, $P$ $<0.001)$, but the estimated glomerular filtration rate values at hemodialysis initiation did not differ. Reasons for temporary CVC use were varied and complex. Problems on the part of healthcare providers, patient behavioral issues, and characteristics of causative kidney disease itself were underlying reasons. Delayed referral to a nephrologist was less frequent than expected (16\%) and the most commonly reported reason (20\%) was that a nephrologist was unable to predict the timing of hemodialysis initiation.

Conclusions: Patients with ESRD should be referred to a nephrologist earlier for AVF creation. However, given the already relatively high rate of hemodialysis initiation with permanent VA in Japan, we considered it surprisingly difficult to further reduce the temporary CVC usage rate in Japan.

Keywords: Arteriovenous fistula, Chronic kidney disease, End-stage renal disease, Nephrology care, Renal replacement therapy, Vascular access

\footnotetext{
* Correspondence: inakaya@chuo-hp.jp

Department of Nephrology and Rheumatology, Iwate Prefectural Central

Hospital, 1-4-1, Ueda, Morioka 020-0066, Japan
}

C C The Author(s). 2021 Open Access This article is licensed under a Creative Commons Attribution 4.0 International License, which permits use, sharing, adaptation, distribution and reproduction in any medium or format, as long as you give appropriate credit to the original author(s) and the source, provide a link to the Creative Commons licence, and indicate if changes were made. The images or other third party material in this article are included in the article's Creative Commons licence, unless indicated otherwise in a credit line to the material. If material is not included in the article's Creative Commons licence and your intended use is not permitted by statutory regulation or exceeds the permitted use, you will need to obtain permission directly from the copyright holder. To view a copy of this licence, visit http://creativecommons.org/licenses/by/4.0/ The Creative Commons Public Domain Dedication waiver (http://creativecommons.org/publicdomain/zero/1.0/) applies to the data made available in this article, unless otherwise stated in a credit line to the data. 


\section{Background}

The number of patients with end-stage renal disease (ESRD) continues to increase, with more than 2 million patients being treated for ESRD worldwide. Of the three renal replacement therapies (RRTs) - hemodialysis (HD), peritoneal dialysis (PD), and kidney transplantation-the largest proportion of patients were receiving HD [1]. Maintaining easily usable vascular access (VA) is critical in patients on HD [2], and this access is greatly influenced by how the initial VA was created [3, 4]. The Japanese Society for Dialysis Therapy (JSDT) clinical guidelines for the construction and repair of VA recommends that the timing of VA creation should be determined based on the clinical symptoms and an estimated glomerular filtration rate (eGFR) of $15 \mathrm{~mL} / \mathrm{min} / 1.73 \mathrm{~m}^{2}$ or less. It also advises predicting the timing of HD initiation from laboratory data and clinical symptoms and creating an arteriovenous fistula (AVF), which is the most common type of VA, at least 2 to 4 weeks before the initial cannulation [5].

These recommendations are based on reports from the JSDT Statistic Survey indicating that the mortality rate was significantly lower in patients who received permanent VA creation 1 to 6 months before HD initiation than in patients who received permanent VA creation between 1 month and the day before HD initiation [6]; likewise, the Dialysis Outcomes and Practice Patterns Study (DOPPS) indicated that receiving the first cannulation within 2 weeks after AVF creation increased VA failure [7]. Additionally, HD initiation with a temporary central venous catheter $(\mathrm{CVC})$ has been reported to have higher costs and longer hospital stays than permanent VA, such as an AVF and arteriovenous graft (AVG) [8].

However, in many cases, HD must be initiated with a temporary $\mathrm{CVC}$ in emergency situations due to delayed consultations or rapidly progressive renal diseases. Additionally, it is difficult to create a well-timed VA because the practical conditions of the community and facility, including the surgeon's intensions and operating room availability, are complicated [2]. By comparison, $80 \%$ of patients in the USA used a temporary CVC at HD initiation [1] whereas this rate was approximately $30 \%$ in Japan $[9,10]$.

Although decreasing the rate of using a temporary CVC at HD introduction would both improve patient prognosis and control healthcare costs by reducing hospitalization time and VA failure, few reports have identified the characteristics and reasons associated with initiating HD with a temporary CVC [11]. We believe that it is possible to identify strategies for reducing the rate of HD initiation with a temporary CVC by determining the reasons for using a temporary CVC at HD initiation. Therefore, this study aimed to clarify the characteristics of patients who were initiated on HD with a temporary CVC and to determine why temporary CVCs were used for reducing the rate of HD initiation with temporary $\mathrm{CVCs}$ in the future. We also surveyed the types of permanent VA created and the time from the creation to the first cannulation of permanent VA.

\section{Methods}

\section{Study design and participants}

This cross-sectional study was conducted in a single facility of the Iwate Prefectural Central Hospital, an acute care general hospital in Japan. The inclusion criteria were as follows: (1) patients who had received permanent VA creation at our department for the introduction of chronic HD between April 2014 and March 2019; (2) 18 years of age or older; and (3) patients in whom HD was initiated by March 2020 at our hospital or two related facilities. Data on 414 patients who met these criteria were extracted from medical records, including surgical registers and dialysis patient lists. The exclusion criteria were as follows: (1) patients who discontinued HD due to recovery of renal function $(n=9),(2)$ patients with a history of PD $(n$ = 8), (3) patients with a history of $\operatorname{HD}(n=1)$, and (4) patients who died during hospitalization following HD initiation $(n=3)$. Thus, 393 patients were enrolled in this study (Fig. 1). We classified the participants into the temporary CVC group or the permanent VA group according to the type of VA at HD initiation and compared their background characteristics.

\section{Clinical parameters}

Clinical data at HD initiation were obtained from medical records and included age, sex, causative disease leading to ESRD, eGFR, serum creatinine levels, and hemoglobin levels. The eGFR was calculated using the three-variable Japanese equation (age, sex, and serum creatinine) [12]. The periods in which the patients received predialysis medical care from nephrologists were categorized as follows: 0-6 months, 6-12 months, 1-2 years, $2-5$ years, or $>5$ years.

\section{Identification of the reasons for using a temporary CVC at HD initiation}

We reviewed medical records to identify why the patients in the temporary CVC group were unable to establish permanent VA at HD initiation. The reason was determined by the concurrence of two nephrologists. We also surveyed the types of first permanent VA created in the CVC group and the time from HD initiation to the creation and first cannulation of permanent VA.

\section{The preferred types of VA in our department}

The most preferred type of VA was radio-cephalic AVF, followed by brachio-cephalic AVF, and ulno-basilic AVF. Several AVGs and subcutaneously fixed superficial 


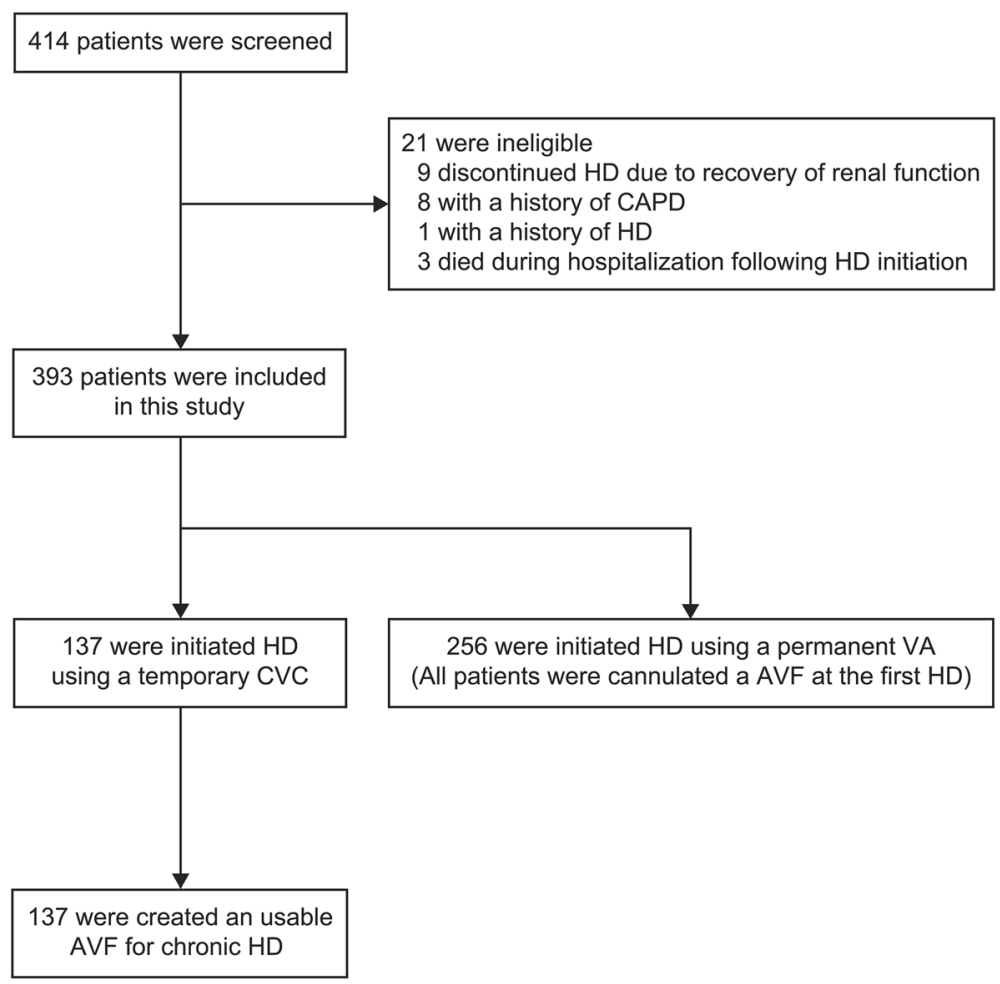

Fig. 1 Screening, exclusion, and classification of the patients

arteries had been created when AVFs could not be created, but no tunneled CVCs were created for several years in our department.

\section{Statistical analysis}

The clinical parameters are shown as the mean \pm standard deviation, median $\left(25^{\text {th }}-75^{\text {th }}\right.$ percentiles $)$, or percentage, as appropriate. The Student's $t$ test and Mann-Whitney $U$ test were used to compare normally and non-normally distributed variables between two groups, respectively. Chi-square tests of independence were used to compare categorical variables between the groups. We also performed a multiple logistic regression analysis to determine the characteristics associated with temporary CVC use in patients with chronic kidney disease (CKD). Statistical analyses were performed using STATA version 15.8 (Stata Corp., College Station, TX). $P<0.05$ was considered statistically significant.

\section{Results}

\section{Patient groups and their first permanent VA}

Of the 393 patients included in this study, HD was initiated using a temporary CVC in 137 (35\%). An AVF was created in all patients in the temporary CVC group during hospitalization following HD initiation, and most patients were transferred to a maintenance dialysis facility after their AVF became usable. All of the remaining 256 patients $(65 \%)$ initiated HD via AVF cannulation. None of the patients in either group had an AVG or tunneled CVC established as their first permanent VA (Fig. 1).

\section{Comparison of patient backgrounds between the temporary CVC group and the permanent VA group}

The patient backgrounds of both groups are shown in Table 1. There was no difference in the mean age between the temporary CVC group $(67.4 \pm 14.4$ years $)$ and the permanent VA group (66.2 \pm 14.7 years). Males accounted for more than two-thirds of the patients in both groups. In the temporary CVC group, $60 \%$ of the patients had been treated by a nephrologist for less than 6 months, and only $35 \%$ had been treated by a nephrologist for more than 1 year. By contrast, in the permanent VA group, $71 \%$ of the patients had received predialysis nephrology care for more than 1 year, and only $18 \%$ had received such care for less than 6 months $(P<0.001)$. In both groups, diabetic kidney disease was the most common cause of ESRD, followed by hypertensive nephrosclerosis and chronic glomerulonephritis. The temporary CVC group comprised more patients with rapidly 
Table 1 Patient characteristics

\begin{tabular}{|c|c|c|c|}
\hline Characteristic & $\begin{array}{l}\text { Temporary CVC } \\
(n=137)\end{array}$ & $\begin{array}{l}\text { Permanent VA } \\
(n=256)\end{array}$ & $P$ value \\
\hline Age (year) & $67.4 \pm 14.4$ & $66.2 \pm 14.7$ & 0.443 \\
\hline Male sex, $n(\%)$ & $92(67.2)$ & $181(70.7)$ & 0.467 \\
\hline Predialysis nephrology care & & & $<0.001^{*}$ \\
\hline$<6$ months, $n(\%)$ & $82(59.9)$ & $45(17.6)$ & \\
\hline 6-12 months, $n(\%)$ & $7(5.1)$ & $29(11.3)$ & \\
\hline $1-2$ years, $n(\%)$ & $13(9.5)$ & $55(21.5)$ & \\
\hline $2-5$ years, $n(\%)$ & $20(14.6)$ & $71(27.7)$ & \\
\hline$>5$ years, $n(\%)$ & $15(10.9)$ & $56(21.9)$ & \\
\hline Kidney disease due to ESRD & & & $0.004^{*}$ \\
\hline $\mathrm{DKD}, n(\%)$ & $45(32.8)$ & $101(39.4)$ & \\
\hline CGN, $n(\%)$ & $22(16.1)$ & $47(18.4)$ & \\
\hline $\mathrm{HN}, n(\%)$ & $32(23.4)$ & $57(22.3)$ & \\
\hline Other CKDs, $n(\%)$ & $20(14.6)$ & $44(17.2)$ & \\
\hline RPGN, $n(\%)$ & $13(9.5)$ & $7(2.7)$ & \\
\hline $\mathrm{AKl}, n(\%)$ & $5(3.6)$ & $0(0.0)$ & \\
\hline Serum creatinine $(\mathrm{mg} / \mathrm{dL})$ & $7.90(6.76-9.69)$ & $8.64(7.28-10.37)$ & $0.017^{*}$ \\
\hline eGFR $\left(\mathrm{mL} / \mathrm{min}\right.$ per $\left.1.73 \mathrm{~m}^{2}\right)$ & $5.34(4.36-6.65)$ & $5.03(4.14-6.06)$ & 0.057 \\
\hline Hemoglobin (g/dL) & $8.7 \pm 1.9$ & $9.3 \pm 1.4$ & $<0.001^{*}$ \\
\hline The time from the creation to the first cannulation of AVFs (days) & $8(6-12)$ & $66(21-139)$ & $<0.001^{*}$ \\
\hline The time from HD initiation to the creation of AVFs (days) & $10(6-17)$ & & \\
\hline The time from HD initiation to the first cannulation of AVFs (days) & $19(14-24)$ & & \\
\hline
\end{tabular}

$A K I$ acute kidney injury, AVF arteriovenous fistula, CKD chronic kidney disease, CGN chronic glomerulonephritis, CVC central venous catheter, DKD diabetic kidney disease, eGFR estimated glomerular filtration rate, ESRD end-stage renal disease, $H D$ hemodialysis, $H N$ hypertensive nephrosclerosis, $R P G N$ rapidly progressive glomerulonephritis, $V A$ vascular access ${ }^{*} P<0.05$

progressive glomerulonephritis (RPGN) and acute kidney injury (AKI) than that in the permanent VA group (9.5\% vs. $2.7 \%$ and $3.6 \%$ vs. $0 \%$, respectively, $P=0.004$ ). A slightly higher percentage of patients in the permanent VA group had diabetic kidney disease than in the temporary CVC group (39\% vs. $33 \%$, respectively). The median serum creatinine level in the temporary CVC group $\left(7.90 \mathrm{mg} / \mathrm{dL}\left(25^{\text {th }}-75^{\text {th }}\right.\right.$ percentiles, $\left.\left.6.76-9.69\right)\right)$ was significantly lower than that in the permanent VA group $(8.64 \mathrm{mg} / \mathrm{dL}(7.28-10.37))(P=0.017)$, but the eGFR values did not differ significantly (5.34 (4.36-6.65) and $5.03(4.14-6.06) \mathrm{mL} / \mathrm{min} / 1.73 \mathrm{~m}^{2}$, respectively $(P=$ $0.057)$ ).

\section{Multiple logistic regression analysis to determine characteristics associated with temporary CVC use in patients with CKD only (Table 2)}

After the exclusion of RPGN and AKI, 368 patients with CKD remained. Age, sex, and kidney disease due to ESRD were not associated with temporary CVC use. Nephrology care had a strong negative association with temporary CVC use, with an odds ratio of 0.643 per one
Table 2 Multiple logistic regression analysis to determine characteristics associated with temporary CVC use in patients with only CKD $(n=368)$

\begin{tabular}{llll}
\hline & Odds ratio & $\mathbf{9 5 \% ~ C l}$ & $\boldsymbol{P}$ value \\
\hline Age (per 1 year) & 0.991 & $(0.973-1.009)$ & 0.327 \\
Sex (Male) & 1.342 & $(0.762-2.366)$ & 0.309 \\
Nephrology care (per 1 category) & 0.643 & $(0.544-0.761)$ & $<0.001^{*}$ \\
eGFR (per 1 ml/min/1.73 m²) & 1.215 & $(1.060-1.394)$ & $0.005^{*}$ \\
Hemoglobin (per 1 g/dL) & 0.794 & $(0.680-0.927)$ & $0.004^{*}$ \\
Kidney disease due to ESRD & & & \\
$\quad$ DKD & 0.619 & $(0.306-1.252)$ & 0.182 \\
$\quad$ CGN & 0.956 & $(0.425-2.149)$ & 0.912 \\
HN & 0.968 & $(0.445-2.109)$ & 0.936 \\
Other CKD & Ref & & \\
\hline
\end{tabular}

CGN chronic glomerulonephritis, CKD chronic kidney disease, CVC central venous catheter, DKD diabetic kidney disease, eGFR estimated glomerular filtration rate, ESRD end-stage renal disease, HN hypertensive nephrosclerosis, 95\% Cl 95\% confidence interval

${ }^{*} P<0.05$ 
category increase $(P<0.001)$. eGFR and hemoglobin levels were also significantly associated with temporary $\mathrm{CVC}$ use, although the causal relationship was unclear.

\section{The time to the first successful cannulation of permanent} VAs

All patients in both groups had an AVF created as their first type of permanent VA. The median time from the AVF creation to the first successful cannulation was significantly shorter in the temporary CVC group than in the permanent VA group ( 8 vs. 66 days, Table $2, P<$ 0.001 ). As shown in Fig. $2 a$, more than $80 \%$ of patients in the temporary CVC group had an AVF successfully punctured within 14 days after AVF creation. Therefore,
$\mathrm{HD}$ at the initial puncture was often performed in a single-needle mode. By contrast, in the permanent VA group, 175 patients $(68 \%)$ were punctured after 30 days, and 46 patients (18\%) were punctured after 6 months (Fig. 2b). In the temporary CVC group, the median time from HD initiation to AVF creation was 10 days (6-17), and the median time from HD initiation to the first successful cannulation of AVF was 19 days (14-24). Thus, more than half of these patients used their temporary CVC for less than 3 weeks.

\section{Reasons for using a temporary CVC at HD initiation}

The reasons for using a temporary CVC at HD initiation were varied and complex. These reasons could be

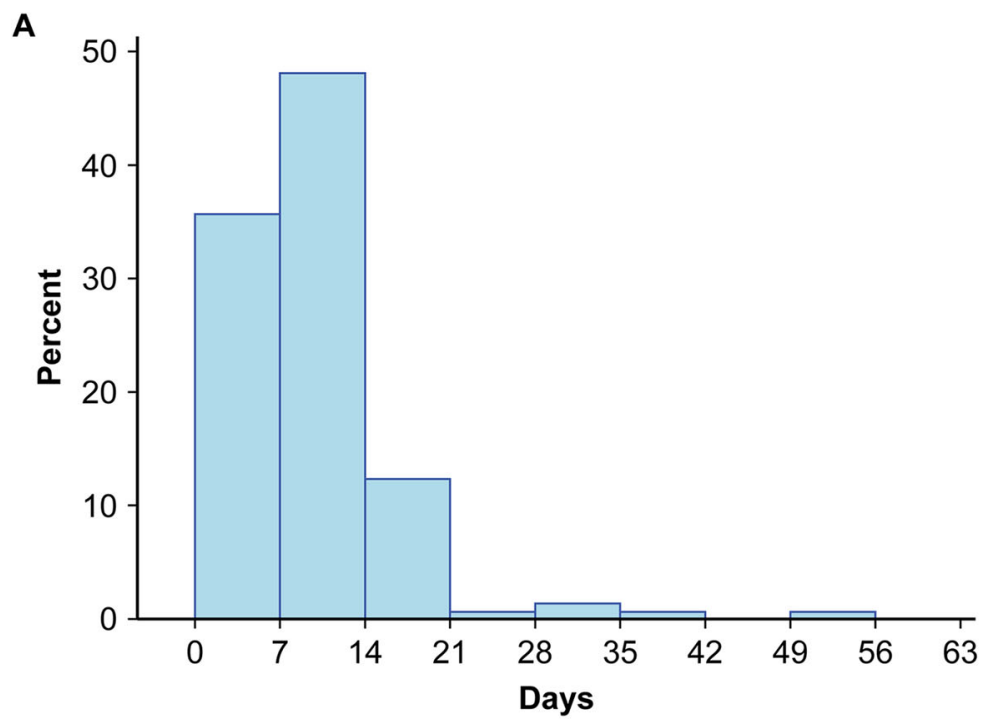

B

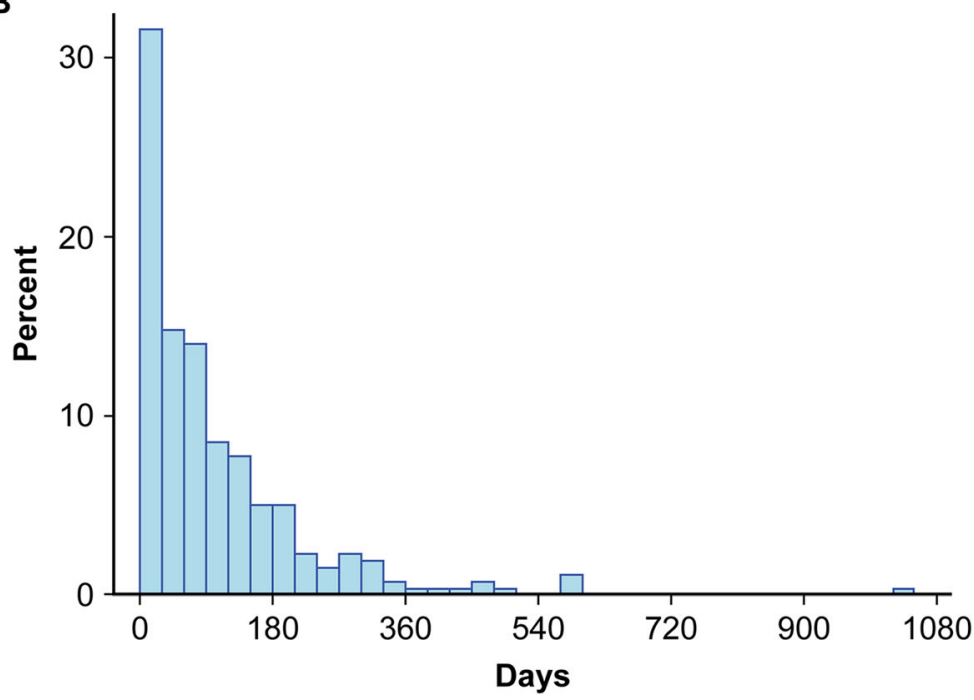

Fig. 2 Histogram (percentage) of the median time from the creation to the first cannulation of AVFs in the temporary CVC group (a) and in the permanent VA group (b). The time for the temporary CVC group was significantly shorter than the time for the permanent VA group (8 vs. 66 days, $P<0.001$ ). More than $80 \%$ of patients in the temporary CVC group had an AVF successfully punctured within 14 days. In contrast, 175 patients (68\%) in the permanent VA group were punctured after 30 days and 46 patients (18\%) were punctured after 6 months 
categorized into nine groups based on the discussion and agreement of two nephrologists and are listed in Table 3 in a descending order of number. The most commonly reported reason (20\%) for using a temporary CVC was the physician being unable to predict the timing of HD initiation despite the patient attending our outpatient clinic for CKD. Delayed referral was reported less frequently than expected (16\%), and a surprisingly high $15 \%$ of patients reported temporary CVC use because of acute exacerbation of kidney function from infection, cardiovascular disease, gastrointestinal disease, etc. Furthermore, $24 \%$ of the reasons for temporary CVC use were related to problems with patients' behavior, including patients who could not reach a decision to create permanent VA for their hesitation and refusal and patients who had not seen a physician. The various characteristics of the causative kidney disease itself were responsible for initiating temporary CVC use in approximately $20 \%$ of such patients, including the patients with RPGN or AKI, patients whose edema was significantly worse, and patients on immunosuppressive therapy for non-diabetic nephrotic syndrome. HD could not be initiated in seven cases (5\%) using a created AVF because of obstruction or underdevelopment. In summary, problems on the part of healthcare providers, patient behavioral issues, and the characteristics of causative kidney disease itself were the underlying reasons for using a temporary CVC at HD initiation.

\section{Patient characteristics stratified by the reasons for using a temporary CVC at HD initiation}

The patient characteristics stratified by the reasons for using a temporary CVC at HD initiation are shown in
Additional file 1 . The patients who had not seen a physician or who had their hospital visits interrupted were the youngest with a mean age of 57 years and had the lowest mean hemoglobin level $(7.3 \mathrm{~g} / \mathrm{dL})$. The highest eGFR was found in patients on immunosuppressive therapy for non-diabetic nephrotic syndrome (median of $13.68 \mathrm{~mL} / \mathrm{min} / 1.73 \mathrm{~m}^{2}$ ). The patients who required urgent HD initiation because of RPGN or AKI comprised a higher percentage of women (53\%); these patients also had the lowest median eGFR $\left(3.96 \mathrm{~mL} / \mathrm{min} / 1.73 \mathrm{~m}^{2}\right)$. Diabetic kidney disease was present in two-thirds of the patients who had initiated HD using temporary CVC due to severe edema. Not surprisingly, the duration of nephrologist treatment was extremely short in the patients who already presented with ESRD at our referral despite attending another outpatient clinic and in patients who had not seen a physician or had interrupted their hospital visits. Thus, the categorized groups had patient characteristics consistent with their respective reasons for temporary $\mathrm{CVC}$ use.

\section{Discussion}

This study in a Japanese acute care general hospital showed that $35 \%$ of patients were introduced to HD with a temporary CVC. The remaining $65 \%$ were introduced to HD with an AVF. The present study also revealed that a usable AVF, which was not an AVG or a tunneled CVC, could be created even in patients of the temporary CVC group during consecutive hospitalization following HD initiation. The most significant difference between the two groups was the duration of predialysis nephrology care. In the temporary CVC group, $60 \%$ of patients had been treated by a nephrologist for less than 6

Table 3 The reasons for HD initiation using a temporary CVC

\begin{tabular}{|c|c|c|}
\hline \multicolumn{2}{|r|}{ Temporary CVC group $(n=137)$} & \multirow{2}{*}{$\begin{array}{l}\boldsymbol{n}(\%) \\
26 \\
(19.0)\end{array}$} \\
\hline 1) & $\begin{array}{l}\text { Although the patients had been attending our outpatient clinic for CKD, their physician failed to predict the timing of their HD } \\
\text { initiation. }\end{array}$ & \\
\hline 2) & Although the patients had been attending another outpatient clinic for CKD, they were already in ESRD at the time of our referral. & $\begin{array}{l}22 \\
(16.1)\end{array}$ \\
\hline 3) & Renal function of the patients was acutely exacerbated by accidental factors, such as infection, CVD, or gastrointestinal disease. & $\begin{array}{l}21 \\
(15.3)\end{array}$ \\
\hline 4) & $\begin{array}{l}\text { Although the patients had been attending our outpatient clinic for CKD and were fully briefed on RRT, they could not make a decision } \\
\text { to create permanent VA for their hesitation and refusal. }\end{array}$ & $\begin{array}{l}17 \\
(12.4)\end{array}$ \\
\hline 5) & $\begin{array}{l}\text { Although the patients had been diagnosed with or suspected of CKD, they had not seen a physician, their hospital visits were } \\
\text { interrupted, and they were already in ESRD at the time of our visit. }\end{array}$ & $\begin{array}{l}16 \\
(11.7)\end{array}$ \\
\hline 6) & HD was urgently initiated in the patients due to RPGN or AKI. & $\begin{array}{l}15 \\
(10.9)\end{array}$ \\
\hline 7) & $\begin{array}{l}\text { HD was initiated in the patients before creating permanent VA because their edema was significantly worse compared to the } \\
\text { worsening of renal function. }\end{array}$ & $9(6.6)$ \\
\hline 8) & Although an AVF was created, their AVF was obstructed or underdeveloped at the time of HD initiation. & $7(5.1)$ \\
\hline () & Although the patients were on immunosuppressive therapy for non-diabetic nephrotic syndrome, they unexpectedly develop & $4(2.9)$ \\
\hline
\end{tabular}

$A K I$ acute kidney injury, AVF arteriovenous fistula, CKD chronic kidney disease, CVC central venous catheter, CVD cardiovascular disease, ESRD end-stage renal disease, $H D$ hemodialysis, $R P G N$ rapidly progressive glomerulonephritis, $R R T$ renal replacement therapy, $V A$ vascular access 
months whereas only $18 \%$ of patients in the permanent VA group had been treated for less than 6 months. Additionally, the time to the first puncture of the AVF was significantly shorter in the temporary CVC group than in the permanent VA group. We found that the reasons for using a temporary CVC at HD initiation were varied and complex. Problems on the part of healthcare providers, patient behavioral issues, and the characteristics of causative kidney disease itself were the underlying reasons.

All patients in this study received an AVF creation as their first permanent type of VA, which had the best prognosis of all types of VA. Considering that approximately $90 \%$ of chronic HD patients in Japan use AVFs and just under $10 \%$ use AVGs [13], this was a very favorable result. The rate of using a temporary CVC at HD initiation was reported to be approximately $20-30 \%$ in Japan [9, 14]. This slightly higher proportion in the present study might be due to the higher rate of RPGN and AKI in patients undergoing HD initiation compared with a Japan nationwide report [15]. Additionally, patients who started HD using a temporary CVC in this study could use an AVF at a median of 19 days, and all patients could use an AVF within 90 days. Compared to the USA, where $68 \%$ of patients were still using a CVC at 90 days after HD initiation [1], we found that AVFs were created promptly in the patients in whom HD was initiated with a temporary CVC in this study. Although various international comparisons have been conducted by the DOPPS, the rate of initiating HD with a temporary CVC should be compared, and a numerical target should be determined in the practice guidelines.

In the present study, AVFs were punctured earlier in the temporary CVC group compared with previous studies. The late referral to a nephrologist and the short duration of predialysis nephrology care were reported to increase the rate of HD initiation with a temporary CVC and lead to early AVF cannulation [16, 17]. Although this study did not investigate patient prognosis, a late referral and HD initiation with a temporary CVC have been reported to be associated with poor prognosis [18, 19], and early AVF puncture has been shown to increase AVF failure $[9,16]$. Thus, particular attention should be paid to AVF failure in patients with a temporary CVC.

To date, few studies have investigated the reasons for using a temporary $\mathrm{CVC}$ at $\mathrm{HD}$ initiation. A previous study more than 20 years ago cited late referral to a nephrologist, late referral to a surgeon, and late decision-making of the patient as the main reasons for using temporary CVCs [11]. However, the primary reason identified in this study was the failure of an attending nephrologist to predict the timing of dialysis initiation; only $16 \%$ of a temporary CVC use was attributed to a late referral. Furthermore, we should emphasize that in our hospital, we, the nephrologists who decided to initiate HD, created the permanent VA ourselves and were easily able to coordinate the date of surgery; thus, there was no delay in referral to the surgeon and few environmental factors that delayed the creation of VA.

It was unclear why AVF could be created in all subjects of the present study, but it might be the result of efforts to create an AVF as much as possible by the nephrologists who were managing VA after HD introduction themselves. Compared with the USA and European countries, the small number of obese patients in Japan might have affected the creation and maturation of AVFs [13]. We have found it difficult to create an AVF in patients with a body mass index of 30 or higher [2]. However, we should not neglect our efforts to create an AVF because an AVF is considered the type of VA with the highest patency rate even in obese patients [20].

In the present study, the duration of predialysis nephrology care was significantly shorter in the temporary CVC group than in the permanent VA group, and duration of predialysis nephrology care was revealed to have a strong association with temporary CVC use in the multiple logistic regression analysis. Although late referral to a nephrologist was not a direct cause for using a temporary CVC at $\mathrm{HD}$ initiation in many patients, the shorter duration of predialysis nephrology care might have had an indirect effect, particularly on the group of patients who could not make the decision to receive permanent VA creation for their hesitation and refusal. $\mathrm{Pa}$ tients' conditions including the type of VA at HD initiation have been reported to influence their prognosis after the initiation of HD [10, 21, 22]. Therefore, an attending physician should refer CKD patients to a nephrologist at least 6 months before the predicted initiation of HD based on the declining eGFR.

We found that there were some patients in whom HD had to be initiated using CVCs in the current medical standard, including some patients with AKI and external factors such as infectious diseases and some with edema so severe that AVF could not be created. This study also revealed that the timing for starting dialysis was unpredictable in many patients despite having visited a nephrologist. Although a previous study discussed how to predict dialysis initiation [23], it may be difficult even for nephrologists to accurately predict the timing for dialysis initiation, and the development of a more accurate tool to predict dialysis initiation may be needed. In addition, there is a large difference in attitudes concerning the creation of an AVF before initiating HD. Opinions differ not only between physicians in different facilities but also between physicians of the same facility. We therefore consider it necessary to spread the significance of AVF creation before initiating HD to many physicians. 
Conversely, $18 \%$ of patients in the permanent VA group started HD at least 6 months after AVF creation, and several patients were initiated HD using a CVC due to an AVF obstruction. In Japan, the mortality rate before beginning dialysis in CKD patients is lower than that in the USA or European countries [24, 25]. However, we must be careful not to create AVFs too early because the increased risk of AVF obstruction has been reported in Japan when AVFs are created too early [26].

The time to the first cannulation from AVF creation in the CVC group was very short in this study compared with other countries [9]. Two main reasons for this difference were considered. First, this may have been performed to reduce the risk of infection. Because the long implantation of temporary CVCs is known to increase the risk of catheter infection [27], shortening the duration of their use might have been a priority in the subjects of this study. Secondly, AVF punctures might have been performed earlier so the patients could be discharged earlier because of the emphasis on shortening the length of hospital stay given Japan's insurance practice. However, since early AVF cannulation may be more problematic in the long term, it is necessary to examine whether early AVF cannulation increases AVF failures after HD initiation in the subjects of this study in the future.

This study has some strengths compared to a similar survey in the past [11], such as collecting more details and performing a comparison with the permanent VA group. However, the results should be understood under some limitations. First, because this study was conducted at a single center in the northeast area of Japan, the findings in this study might not be generalizable to patients outside of Japan under different healthcare systems. Furthermore, more than $90 \%$ of patients with ESRD chose HD as their first RRT in our department where this study was conducted. In situations where more CKD patients choose PD or preemptive kidney transplantation as their first RRT, the number of patients who initiated HD with a permanent VA may decrease and the using rate of temporary CVC may increase. Second, because this was a cross-sectional study, we could not clarify the patients' survival and VA prognosis in the temporary CVC group.

\section{Conclusions}

This study revealed the characteristics and reasons why patients were initiated on HD with a temporary CVC. ESRD patients should be referred to a nephrologist earlier for AVF creation. However, we found that the reasons for using a temporary CVC at HD initiation were varied and complex. Given the already relatively high rate of HD initiation with permanent VA in Japan, it may be surprisingly difficult to further reduce the rate of temporary $\mathrm{CVC}$ use at $\mathrm{HD}$ initiation by changing the behavior of healthcare providers and CKD patients. Future international prospective studies are necessary to reveal more details of the reasons for using a temporary CVC at HD initiation worldwide, and we hope the rate of temporary CVC use is gradually reduced as much as possible in the future.

\section{Supplementary Information}

The online version contains supplementary material available at https://doi. org/10.1186/s41100-021-00318-y.

Additional file 1. Patient characteristics stratified by the reasons for $\mathrm{HD}$ initiation with a temporary CVC.

\section{Abbreviations}

AKI: Acute kidney injury; AVF: Arteriovenous fistula; AVG: Arteriovenous graft; CKD: Chronic kidney disease; CVC: Central venous catheter; DOPPS: Dialysis

Outcomes and Practice Patterns Study; eGFR: Estimated glomerular filtration rate; ESRD: End-stage renal disease; HD: Hemodialysis; JSDT: Japan Society for Dialysis Therapy; PD: Peritoneal dialysis; RPGN: Rapidly progressive glomerulonephritis; RRT: Renal replacement therapy; VA: Vascular access

\section{Acknowledgements}

This work originated from the Iwate Prefectural Central Hospital.

\section{Authors' contributions}

Conceived and designed the experiments: IN, TG, and YN; performed the experiments: IN, TG, YN, KY, JO, YT, MM, SI, HI, and YM; analyzed the data: IN and TG; wrote the paper: IN, TG, and JS. The author(s) read and approved the final manuscript.

\section{Funding}

This study was not supported by any grant or sponsor.

\section{Availability of data and materials}

The datasets used and/or analyzed during the present study are available from the corresponding author upon reasonable request.

\section{Ethics approval and consent to participate}

The Ethics Committee of Iwate Prefectural Central Hospital approved this study, and it was conducted in accordance with the ethical principles of the Declaration of Helsinki. We did not obtain written informed consent from the patients because the ethical guidelines for epidemiological research in Japan do not require informed consent for a cross-sectional study using only existing medical records.

Consent for publication

Not applicable.

\section{Competing interests}

The authors declare that they have no competing interests.

Received: 19 September 2020 Accepted: 4 January 2021 Published online: 14 January 2021

References

1. Saran R, Robinson B, Abbott KC, Agodoa LYC, Bhave N, Bragg-Gresham J, et al. Epidemiology of kidney disease in the United States. Am J Kidney Dis. 2018;71(3 Suppl 1):A7

2. Woo K, Lok CE. New insights into dialysis vascular access: what is the optimal vascular access type and timing of access creation in CKD and dialysis patients? Clin J Am Soc Nephrol. 2016;11:1487-94.

3. Lok CE, Huber TS, Lee T, Shenoy S, Yevzlin AS, Abreo K, et al. KDOQI clinical practice guideline for vascular access: 2019 update. Am J Kidney Dis. 2020; 75:S1-S164.

4. Robinson BM, Akizawa T, Jager KJ, Kerr PG, Saran R, Pisoni RL. Factors affecting outcomes in patients reaching end-stage kidney disease 
worldwide: differences in access to renal replacement therapy, modality use, and haemodialysis practices. Lancet. 2016;388:294-306.

5. Kukita K, Ohira S, Amano I, Naito H, Azuma N, lkeda K, et al. 2011 Update Japanese Society for Dialysis Therapy guidelines of vascular access construction and repair for chronic hemodialysis. Ther Apher Dial. 2015; 19(Suppl 1):1-39.

6. Nakai S, Masakane I, Shigematsu T, Hamano T, Yamagata K, Watanabe Y, et al. An overview of regular dialysis treatment in Japan (as of 31 December 2007). Ther Apher Dial. 2009;13:457-504.

7. Allon M, Imrey PB, Cheung AK, Radeva M, Alpers CE, Beck GJ, et al. Relationships between clinical processes and arteriovenous fistula cannulation and maturation: a multicenter prospective cohort study. Am J Kidney Dis. 2018;71:677-89.

8. Wu LC, Lin MY, Hsieh CC, Chiu HC, Mau LW, Chiu YW, et al. Planned creation of vascular access saves medical expenses for incident dialysis patients. Kaohsiung J Med Sci. 2009;25:521-9.

9. Rayner HC, Pisoni RL, Gillespie BW, Goodkin DA, Akiba T, Akizawa T, et al. Creation, cannulation and survival of arteriovenous fistulae: data from the Dialysis Outcomes and Practice Patterns Study. Kidney Int. 2003;63:323-30.

10. Higuchi S, Nakaya I, Yoshikawa K, Chikamatsu Y, Sada KE, Yamamoto S, et al. Potential benefit associated with delaying initiation of hemodialysis in a Japanese cohort. Kidney Int Rep. 2017;2:594-602.

11. Chesser AM, Baker LR. Temporary vascular access for first dialysis is common, undesirable and usually avoidable. Clin Nephrol. 1999;51:228-32.

12. Matsuo S, Imai E, Horio M, Yasuda Y, Tomita K, Nitta K, et al. Revised equations for estimated GFR from serum creatinine in Japan. Am J Kidney Dis. 2009:53:982-92.

13. Pisoni RL, Zepel L, Port FK, Robinson BM. Trends in US vascular access use, patient preferences, and related practices: an update from the us DOPPS practice monitor with international comparisons. Am J Kidney Dis. 2015;65: 905-15.

14. Ozeki T, Shimizu H, Fujita Y, Inaguma D, Maruyama S, Ohyama Y, et al. The type of vascular access and the incidence of mortality in Japanese dialysis patients. Intern Med. 2017;56:481-5.

15. Masakane I, Nakai S, Ogata S, Kimata N, Hanafusa N, Hamano T, et al. An overview of regular dialysis treatment in Japan (as of 31 December 2013). Ther Apher Dial. 2015;19:540-74.

16. Ravani P, Brunori G, Mandolfo S, Cancarini G, Imbasciati E, Marcelli D, et al. Cardiovascular comorbidity and late referral impact arteriovenous fistula survival: a prospective multicenter study. J Am Soc Nephrol. 2004;15:204-9.

17. Gonçalves EA, Andreoli MC, Watanabe R, Freitas MC, Pedrosa AC, Manfredi $\mathrm{SR}$, et al. Effect of temporary catheter and late referral on hospitalization and mortality during the first year of hemodialysis treatment. Artif Organs. 2004;28:1043-9.

18. Yamagata K, Nakai S, Masakane I, Hanafusa N, Iseki K, Tsubakihara Y. Ideal timing and predialysis nephrology care duration for dialysis initiation: from analysis of Japanese dialysis initiation survey. Ther Apher Dial. 2012;16:54-62.

19. Lorenzo V, Martn M, Rufino M, Hernandez D, Torres A, Ayus JC. Predialysis nephrologic care and a functioning arteriovenous fistula at entry are associated with better survival in incident hemodialysis patients: an observational cohort study. Am J Kidney Dis. 2004;43:999-1007.

20. Okawa T, Murakami M, Yamada R, Tanaka S, Mori K, Mori N. One-stage operation for superficialization of native radio-cephalic fistula in obese patients. J Vasc Access. 2019;20(Suppl 1):45-9.

21. de Mutsert R, Grootendorst DC, Boeschoten EW, Brandts H, van Manen JG, Krediet RT, et al. Subjective global assessment of nutritional status is strongly associated with mortality in chronic dialysis patients. Am J Clin Nutr. 2009:89:787-93.

22. Ocak G, Rotmans Jl, Vossen CY, Rosendaal FR, Krediet RT, Boeschoten EW, et al. Type of arteriovenous vascular access and association with patency and mortality. BMC Nephrol. 2013;14:79.

23. Tangri N, Stevens LA, Griffith J, Tighiouart H, Djurdjev O, Naimark D, et al. A predictive model for progression of chronic kidney disease to kidney failure. JAMA. 2011;305:1553-9.

24. Nakayama M, Sato T, Sato H, Yamaguchi Y, Obara K, Kurihara I, et al. Different clinical outcomes for cardiovascular events and mortality in chronic kidney disease according to underlying renal disease: the Gonryo study. Clin Exp Nephrol. 2010;14:333-9.

25. Keith DS, Nichols GA, Gullion CM, Brown JB, Smith DH. Longitudinal followup and outcomes among a population with chronic kidney disease in a large managed care organization. Arch Intern Med. 2004;164:659-63.
26. Miyamoto M, Kurita N, Suemitsu K, Murakami M. Fistula and survival outcomes after fistula creation among predialysis chronic kidney disease stage 5 patients. Am J Nephrol. 2017:45:356-64.

27. Naumovic RT, Jovanovic DB, Djukanovic L. Temporary vascular catheters for hemodialysis: a 3-year prospective study. Int J Artif Organs. 2004;27:848-54.

\section{Publisher's Note}

Springer Nature remains neutral with regard to jurisdictional claims in published maps and institutional affiliations.

\section{Ready to submit your research? Choose BMC and benefit from:}

- fast, convenient online submission

- thorough peer review by experienced researchers in your field

- rapid publication on acceptance

- support for research data, including large and complex data types

- gold Open Access which fosters wider collaboration and increased citations

- maximum visibility for your research: over $100 \mathrm{M}$ website views per year

At BMC, research is always in progress.

Learn more biomedcentral.com/submissions 